\title{
An automated framework for high-throughput predictions of NMR chemical shifts within liquid solutions
}

\author{
Rasha Atwi \\ Stony Brook University \\ Ying Chen \\ Pacific Northwest National Laboratory \\ Kee Sung Han \\ Pacific Northwest National Laboratory \\ Karl Mueller \\ Pacific Northwest National Laboratory \\ Vijayakumar Murugesan \\ Pacific Northwest National Laboratory \\ Nav Nidhi Rajput ( $\square$ navnidhi.rajput@stonybrook.edu ) \\ Stony Brook University
}

Article

Keywords: nuclear magnetic resonance, classical molecular dynamics, chemical shifts

Posted Date: October 4th, 2021

DOl: https://doi.org/10.21203/rs.3.rs-893249/v1

License: (1) This work is licensed under a Creative Commons Attribution 4.0 International License.

Read Full License

Version of Record: A version of this preprint was published at Nature Computational Science on February 1st, 2022. See the published version at https://doi.org/10.1038/s43588-022-00200-9. 

NMR chemical shifts within liquid solutions

3 Rasha Atwi ${ }^{1}$, Ying $\mathrm{Chen}^{2}$, Kee Sung $\mathrm{Han}^{2}$, Karl T. Mueller ${ }^{2}$, Vijaykumar Murugesan ${ }^{2}$, Nav Nidhi Rajput*1

$5 \quad{ }^{1}$ Department of Materials Science and Chemical Engineering, Stony Brook University, Stony Brook, New York 11794, United States

Richland, WA 99352, USA

$20 *$ To whom correspondence should be addressed:

21 Nav Nidhi Rajput; Email: navnidhi.rajput@stonybrook.edu 


\section{ABSTRACT}

23 Identifying stable speciation in multicomponent liquid solutions is of fundamental importance to

24 areas ranging from electrochemistry to organic chemistry and biomolecular systems. However,

25 elucidating this complex solvation environment is a daunting task even when using advanced

26 experimental and computational techniques. Here, we introduce a fully automated, high-

27 throughput computational framework for the accurate and robust prediction of stable species

28 present in liquid solutions by computing the nuclear magnetic resonance (NMR) chemical shifts

29 of molecules. The framework automatically extracts and categorizes hundreds of thousands of

30 atomic clusters from classical molecular dynamics (CMD) simulations to identify the most stable

31 speciation in the solution and calculate their NMR chemical shifts via DFT calculations.

32 Additionally, the framework creates an output database of computed chemical shifts for liquid

33 solutions across a wide chemical and parameter space. This task can be infeasible experimentally

34 and challenging using conventional computational methods. To demonstrate the capabilities of our

35 framework, we compare our computational results to experimental measurements for a complex

36 test case of magnesium bis(trifluoromethanesulfonyl)imide $\mathrm{Mg}(\mathrm{TFSI})_{2}$ salt in dimethoxyethane

37 (DME) solvent, which is a common electrolyte system for Mg-based batteries. Our extensive

38 benchmarking and analysis of the $\mathrm{Mg}^{2+}$ solvation structural evolutions reveal critical factors such

39 as the effect of force field parameters that influence the accuracy of NMR chemical shift

40 predictions in liquid solutions. Furthermore, we show how the framework reduces the efforts of

41 performing and managing over $300{ }^{13} \mathrm{C}$ and $600{ }^{1} \mathrm{H}$ DFT chemical shift predictions to a single

42 submission procedure. By enabling more efficient and accurate high-throughput computations of

43 NMR chemical shifts, our approach can accelerate theory-guided design of liquid solutions for 44 various applications. 
INTRODUCTION

46 Liquid solutions are critical components of various chemical, materials science, engineering,

47 and biological applications such as batteries ${ }^{1-3}$, fuel ${ }^{4}$, food industry ${ }^{5}$, and drug discovery ${ }^{6,7}$.

48 Optimizing the performance of these technologies requires taking into careful account transport

49 and structural features, along with the thermodynamic stability of chemical compounds comprising

50 the solution. More specifically, developing a fundamental understanding of the correlations

51 between functional properties and the underlying atomistic interactions is necessary for advancing

52 the rational design of liquid solutions. In this regard, nuclear magnetic resonance (NMR)

53 spectroscopy stands out as a powerful and widespread technique for studying the 3D organization

54 of matter and associated structural and dynamical properties ${ }^{8-10}$. Over the years, technological

55 advances in NMR spectroscopy have significantly improved the operational ease and spectral

56 resolutions obtainable from non-traditional nuclei (such as ${ }^{17} \mathrm{O},{ }^{25} \mathrm{Mg}$, etc.), leading to a

57 comprehensive and atomistic view of liquid solutions ${ }^{11,12}$. However, NMR spectroscopy is limited

58 by the temporal scale and low sensitivity, making it difficult to speciate structural patterns that are

59 often driven by electrostatic interactions, reactivity, temperature, compositional variance, and

60 pressure $^{13-15}$

61 In such complex scenarios, computational NMR studies are necessary to decipher experimental

62 results and better understand different chemical and physical effects whose interplay determines

63 the overall spectrum. For example, ab-initio molecular dynamics (AIMD) simulations have been

64 used to capture the structural evolutions and associated chemical shifts ${ }^{16-18}$. However, the

65 computational cost associated with large systems (>100 atoms) and simulation time scales $(\sim 10$

66 ps) imposes severe restrictions for tests of liquid solutions across a wide chemical space. Density

67 functional theory (DFT) calculations have also provided valuable insights into chemical shift 
68 trends $^{8,19,20}$. However, they fail to fingerprint the temporal evolution of solvation structures under

69 exogenous (temperature and pressure) and endogenous ( $\mathrm{pH}$ and composition) conditions. In

70 addition, gaps in knowledge between systems examined in-situ or ex-situ and those modeled in

71 silico still exist. For example, NMR DFT studies are often focused on singular phenomena, e.g.,

72 magnetic shielding tensor. Recently, an automated framework ${ }^{21}$ and a machine learning based

73 approach ${ }^{22}$ were implemented to predict the ${ }^{13} \mathrm{C} /{ }^{1} \mathrm{H}$ NMR chemical shift for organic molecules.

74 However, a generalized approach to identify complexes in multi-component solutions and

75 accurately predict NMR chemical shift especially for non-traditional nuclei remains a great

76 challenge. On the other hand, NMR experiments can reveal much more information about the

77 chemical system, such as details of chemical exchange, correlation times or energetics for

78 rotational and translational dynamics, etc. Even for the singular focus on chemical shift

79 calculations, the possible molecular structure(s) are built manually based on chemical intuition,

80 trial and error, and/or results reported in the literature $20,23,24$. This approach of providing the initial

81 guesses is fraught with bias, is time-consuming, can be challenging to automate fully, and leaves

82 behind many persistent metastable configurations of fundamental importance for interpreting

83 experimental results. To overcome these challenges, we designed an automated computational

84 framework that allows accurate prediction of NMR chemical shifts even in complex

85 multicomponent liquid solutions and guide experiments to identify stable speciation in the 86 solution.

87 The paper is composed of two sections. First, we discuss the details of our high-fidelity and

88 robust computational tool that seamlessly integrates classical molecular dynamics (CMD)

89 simulations with DFT calculations through force field generation and information flow between

90 the two length scales. The tool automates the entire process, starting from sampling hundreds of 
91 thousands of possible configurations in solute-solvent systems to identifying the most stable

92 configurations and predicting and storing their NMR chemical shifts in a database. To the best of

93 our knowledge, an automatic derivation of NMR chemical shifts with explicit solvation has not

94 yet been implemented in any software infrastructure. Although the developed tool is general

95 enough to be applied to any liquid solution, we consider magnesium

96 bis(trifluoromethanesulfonyl)imide $\mathrm{Mg}(\mathrm{TFSI})_{2}$ salt in dimethoxyethane (DME) solvent as an

97 illustrative example. The chosen electrolyte formulation has received considerable attention in

98 battery literature but reported findings regarding the speciation and the exact solvation structure

99 of the $\mathrm{Mg}$ cation are under contention. More specifically, experimental work reported the

100 formation of solvent separated ion pairs (SSIPs), while contact ion pairs (CIPs) were observed in

101 previous computational results ${ }^{14,23,25,26}$. A comprehensive molecular level understanding of the

102 speciation present in the solution can allow tuning the chemical structure to control the stability,

103 solubility, structural, and dynamical properties of liquid solutions. We note that we chose a system

104 in which complexities in the solvation phenomena arise due to the multivalent nature of the cation,

105 providing an example to demonstrate that the developed framework can be applied to other simpler

106 systems. We report a detailed comparison between computed and experimental NMR chemical

107 shifts for ${ }^{25} \mathrm{Mg},{ }^{13} \mathrm{C}$, and ${ }^{1} \mathrm{H}$ nuclei in this electrolyte. We also demonstrate the high-throughput

108 capability of the workflow by accurately predicting more than $300{ }^{13} \mathrm{C}$ and $600{ }^{1} \mathrm{H}$ NMR chemical

109 shifts from a set of 100 organic molecules from the $\mathrm{SDBS}^{27}$ database and a previous experimental

110 study $^{28}$. In the second section, we address the fundamental challenge of how to accurately predict

111 NMR chemical shift of liquid solutions by associating the framework with a benchmarking study.

112 This study reveals several factors such as the choice of force field parameters that affect the

113 accuracy of predicted chemical shifts, which can be employed by a number of research 
114 communities by increasing the accessibility to DFT-based chemical shifts for a wide variety of

115 structures and liquid systems.

\section{RESULTS AND DISCUSSION}

\section{Overview of the automated framework}

118 We construct an NMR computational framework using MISPR (Molecular Informatics for

119 Structure-Property-Relationship), our high-throughput and scalable infrastructure that allows

120 automatic handling of thousands of computational materials science simulations and multiple

121 systems with a strong focus on data provenance. MISPR automates many computational tasks that

122 are typically performed manually. Its functionalities span from processing and manipulating

123 molecular structures, preparing and executing DFT and CMD simulations on supercomputing

124 resources, parsing and analyzing output data, and creating output databases that organize the

125 results from individual calculations. To manage the heterogeneous data that DFT and CMD

126 workflows output and allow for flexible and complex queries, MISPR employs MongoDB ${ }^{29}$ for

127 data storage. MongoDB is a document-oriented NoSQL database that stores data as JSON-

128 formatted documents with flexible schema. A unique feature of MISPR is that it allows seamless

129 and automated integration of DFT calculations with CMD simulations to capture structural and

130 dynamical phenomena that span over wide spatial and temporal scales. It contains multiple preset

131 DFT and CMD workflow templates that, from the outside, the user only needs to call in a single

132 Python script with minimal required inputs (e.g., molecular structure, the size and geometry of the

133 system for CMD simulations, etc.) to generate and run a comprehensive workflow. We built

134 MISPR on top of base libraries developed by the Materials Project, namely: (1) pymatgen ${ }^{30}$ for

135 structure representation and input/output files generation and handling, (2) FireWorks ${ }^{31}$ for

136 managing workflows over computing resources, and (3) custodian $^{32}$ for monitoring inevitable 
137 errors during simulations and applying on-the-fly fixes. At the backend, MISPR uses Gaussian ${ }^{33}$ 138 electronic structure software for DFT calculations and LAMMPS ${ }^{34}$ (https://www.lammps.org/) 139 open-source code for CMD simulations. Examples of implemented DFT workflows include 140 calculating binding energy, redox potentials, and bond dissociation energy. CMD workflows in 141 MISPR allow executing CMD simulations in various ensembles and analyzing collected 142 trajectories for structural and dynamical properties. Force field parameters and derived properties 143 are saved in their collections with auxiliary information like molecular metadata (e.g., InChI 144 representation, chemical formula, etc.) and input parameters, making it easy to reproduce and 145 query computational results. More details about the MISPR infrastructure will be the subject of a 146 future publication.

147 The framework designed for automatic NMR chemical shift calculations in liquid solutions is 148 outlined in Fig 1. The framework takes as input the structures of molecules comprising a liquid 149 solution of interest. Many molecule formats are supported (e.g., XYZ file, PDB file, pymatgen 150 molecule object, Gaussian output, etc.) via the OpenBabel ${ }^{35}$ and pymatgen libraries. Besides these 151 formats, the framework can take query criteria to retrieve previously optimized structures from the 152 database. It can also derive a structure on the fly by either attaching a functional group or linking 153 two structures at a specific binding site. Next, the framework runs an electrostatic partial charges 154 (ESP) workflow that first converts the input structure formats to pymatgen molecule objects. The 155 ESP workflow uses this molecule object to generate a Gaussian input file with input parameters 156 specified as optional inputs to the workflow. The workflow uses default values if these parameters 157 are not provided. It then runs three sequential steps: (1) a DFT geometry optimization, (2) a 158 vibrational frequency calculation to ensure that there are no imaginary frequencies, and (3) a 
159 population analysis to assign atomic charges. The framework executes the ESP workflow for each 160 component of the liquid solution.

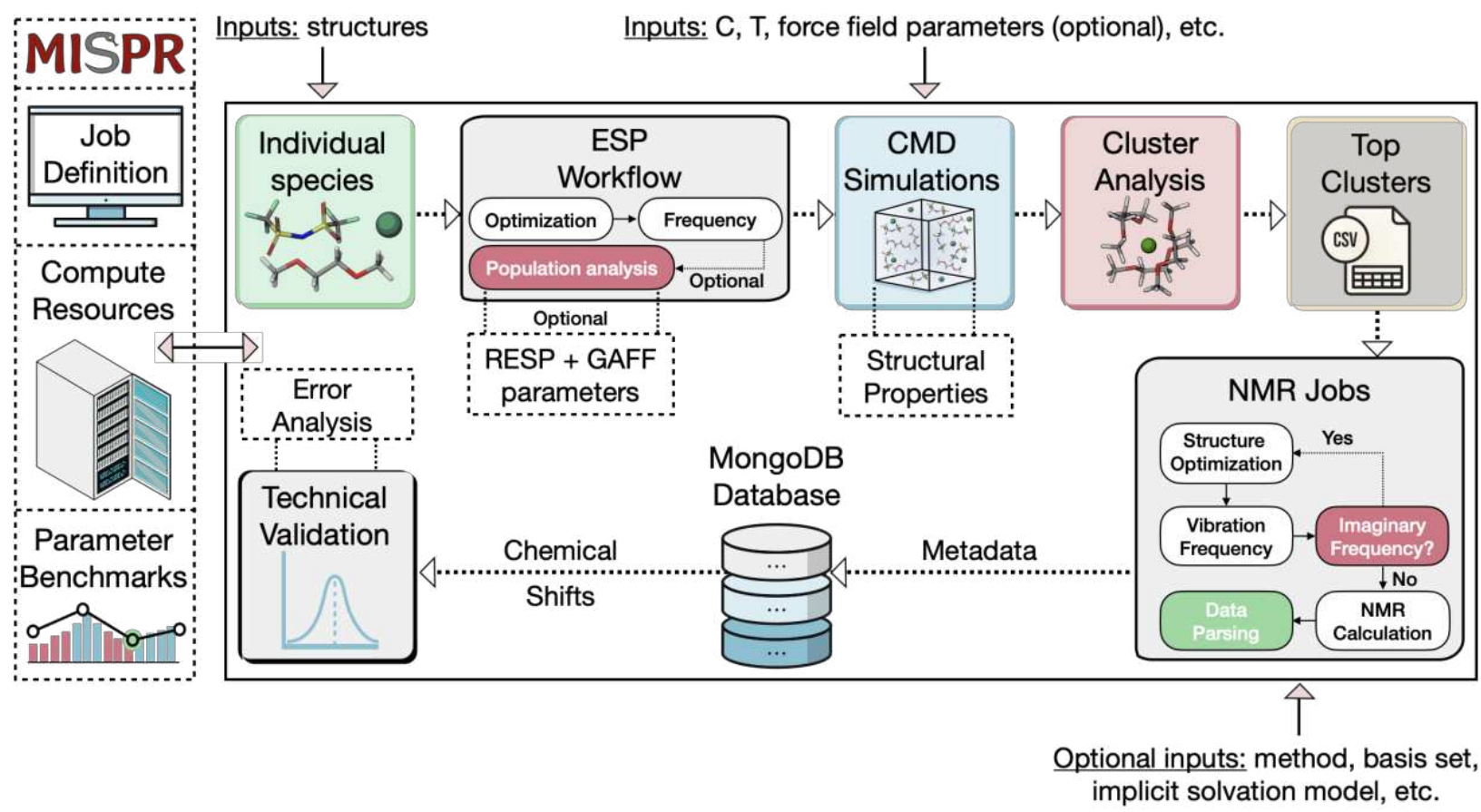

Fig. 1 Scheme of the computational framework used to calculate NMR chemical shifts in solution as implemented in the MISPR high-throughput infrastructure

We note that the framework is general enough to be applied to various complex liquid solutions

162 at different conditions (e.g., concentration, temperature, pressure, etc.). It requires, at minimum,

163 the concentration of species in the solution and the size and geometry of the system box to prepare

164 the multicomponent system for CMD simulations. One of the most challenging aspects of running 165 automated CMD simulations is selecting or generating accurate force field parameters. By default,

166 the framework uses the output of the ESP workflow to derive the general amber force field $167(\mathrm{GAFF})^{36}$ parameters for each species. The framework also supports other force fields allowing 168 the user to test different physical models for a specific application or system. In this case, the user 169 may input the force field parameters to the framework in the form of a Python dictionary or retrieve 170 them from our in-house database. We note that the user may bypass the ESP workflow if the ESP 171 charges have been previously calculated or other force fields are directly provided. The framework 
172 then passes the optimized geometries, force field parameters, concentrations, and information 173 about the geometry of the simulation box (e.g., lengths, shape, etc.) to the next step to build the 174 system for LAMMPS simulations. Following this, the framework runs a CMD workflow to 175 generate time trajectories of atomic positions and velocities. Configurations for common CMD 176 procedures are encoded in a set of protocols that can be used directly or altered to run any series 177 of LAMMPS calculations according to the user's needs. The default CMD configuration involves 178 energy minimization, NPT equilibration at the desired temperature and pressure, melting and 179 quenching, and NVT production runs.

180 The framework then uses the generated LAMMPS trajectory files to compute the radial 181 distribution function (RDF) between all possible pairs of particle types in the system or specific 182 pairs specified as inputs. The RDF module is part of a standalone in-house suite of Python tools 183 that we developed to extract a range of structural and dynamical properties from LAMMPS 184 trajectory and output files. The RDF defines the probability of finding a particle at a distance $r$ 185 from another particle. More details about the RDF calculations are provided in the section 1 of the 186 SI.

187 Sampling solvation structures from the CMD step is a key component of the NMR framework.

188 Traditional NMR calculations are relatively inefficient at constructing initial guesses for molecular 189 structures. Building molecular structures by manually placing a number of molecules in the 190 solvation shell of the particle of interest is extremely time consuming $20,23,24$. In contrast, our 191 framework passes the computed RDF from the previous step to perform sampling of the first 192 solvation shell of a particle of interest in a straightforward and automated manner. In the 193 framework, the first solvation shell is defined by the cutoff distance $r_{\text {min }}$, corresponding to the 194 position of the first minimum after the main peak of the RDF between the particle of interest and 
195 other coordinating particles in the solution. In the default operation of the framework, $r_{\min }$ is 196 automatically extracted from the RDF, but the user may override this by providing $r_{\min }$ as an 197 optional input. Thus, a cluster representing the solvation structure is defined as the group of species 198 within $r_{\min }$ of the particle. By ensemble averaging hundreds of thousands of clusters, we obtain a 199 distribution of clusters corresponding to all the possible chemical environments surrounding the 200 particle of interest in the solution.

201 Next, the framework categorizes the extracted clusters into unique configurations based on the 202 type and number of species surrounding the particle and their mode of coordination. Then, it 203 calculates the probability of each configuration as the ratio of the number of clusters that belong 204 to a specific configuration to the total number of extracted clusters. Configurations with the highest 205 probability of occurrence correspond to persistent metastable solvation structures in the solution. 206 By default, the framework selects the top configurations whose probabilities sum to more than $20790 \%$ of the total number of extracted clusters, but the user may also select the configurations as 208 needed. The selection of the configurations is done to reduce the number of required DFT 209 calculations and their associated computational cost. It is also important to select a representative 210 cluster from each configuration since it is common that thousands of clusters with subtle 211 geometrical differences (e.g., bond lengths, orientation, etc.) belong to the same configuration. To 212 this end, the framework performs a local minimization procedure on all the clusters from the 213 selected top configurations using the MMFF94s force field ${ }^{37}$ as implemented in the RDKit 214 library ${ }^{38}$. The framework then feeds the lowest-energy conformer of each configuration to an NMR

215 DFT workflow. We note that it would be infeasible to manually generate and categorize this large 216 number of structural files and account for all the possible solvation structures using conventional 
217 methods that rely on chemical intuition. This task is especially challenging for chemical systems

218 that have not been previously explored in detail.

219 The NMR workflow relaxes the CMD clusters selected from the previous step, performs a 220 vibrational frequency analysis, and calculates the magnetic shielding tensor on each atom if a true 221 potential energy surface (PES) minimum is reached. The framework by default uses the $222 \omega \mathrm{B} 97 \mathrm{X}^{39} / \mathrm{def} 2-\mathrm{TZVP}$ level of theory for performing these three sequential DFT steps. Switching 223 the functional, basis set, and other Gaussian input parameters (e.g., solvation model, numerical 224 and algorithmic parameters, etc.) is straightforward and requires the user to input them in the form 225 of a Python dictionary to the framework. The framework then performs an analysis step that stores 226 the calculation results in an NMR collection in the database or a local JSON file with all the 227 necessary metadata for future reference. Creating a local file allows the user to check outputs 228 quickly, retrieve data without accessing the database, and exchange data with other parties. An 229 example of the structure of an NMR document is shown in Fig S1. Finally, results from the 230 computational framework are compared to experimental NMR spectra to elucidate the solvation 231 structures.

232 In the NMR workflow, a series of convergence checks are performed to ensure the results are 233 as reliable as possible. For example, we implemented checks for normal termination of DFT 234 calculations and automatic inspection of the 3D structure resulting from optimization to confirm 235 connectivity matches the input structure. Once each step of the NMR workflow has terminated, 236 the output file is parsed for errors. An automatic error correction process is employed through 237 well-defined rules via the custodian package if an error is detected. If possible, the error handler 238 applies the appropriate remedy, generally by modifying the input parameters, writing a new 239 Gaussian input file, and restarting the calculation. If no remedy has been implemented for a 
240 particular error or the error handler cannot interpret the encountered error, the calculation is

241 allowed to fail. The error handler improves the success rate of the calculations without relying on

242 human intervention, which would be impossible for handling large computational investigations.

243 Examples of the errors addressed are SCF failure, geometry optimization convergence, error in

244 internal coordinates, and exceeded wall time limit.

245 The framework takes solvent effects into account by two approaches. It uses an explicit 246 approach where several solvent molecules surrounding the species are correctly placed in its first

247 solvation shell since the geometries are extracted directly from CMD simulations. Second, it 248 approximates bulk solvent effects using a dielectric continuum model. This approach allows 249 incorporating a thermodynamically stable and realistic chemical environment of species compared 250 to the traditional approach, which relies on either implicit solvent models or manual prediction of 251 the possible solvation structures. Since multiple configurations are considered, collected data result 252 in various chemical shifts corresponding to different chemical environments experienced by the 253 nucleus of interest. Therefore, predictions from this approach can be compared and fitted to the 254 entire experimental NMR peak rather than just matching the peak center, especially when peak 255 broadening occurs due to distribution of chemical shifts or intermediate exchange dynamics in 256 solutions.

257 Components of the NMR framework presented in Fig 1 can be decoupled according to the 258 needs of the user. For example, we used the NMR workflow as a standalone code to compute the $259{ }^{13} \mathrm{C}$ and ${ }^{1} \mathrm{H}$ chemical shifts for a set of 100 organic molecules. Detailed information about the 260 library is provided in Table S1. The calculations were performed in a chloroform solvent at the $261 \omega \mathrm{B} 97 \mathrm{X} / \mathrm{def} 2-\mathrm{TZVP}$ level of theory and referenced to tetramethylsilane (TMS). The code snippet 262 in Fig S2 demonstrates how to submit these calculations starting from structures defined in the 
263 XYZ file format. Upon submitting the script, the calculations were added to a FireWorks database

264 and subsequently executed over computing resources. The workflow generated and managed over

265600 input and output files and inserted more than $300{ }^{13} \mathrm{C}$ and $600{ }^{1} \mathrm{H}$ chemical shifts into the

266 database via a simple one-shot script. We compared our predictions to experimental data from the

267 SDBS $^{27}$ database and a previous study ${ }^{28}$. Fig S3 and Fig S4 show parity plots of the computed

268 chemical shifts and their associated error distribution, respectively. A good correlation is observed

269 between the workflow output and the experimental data with only minor deviations from the fitted

270 line. This example demonstrates how our high-throughput approach may be adapted for the

271 determination of accurate NMR chemical shifts.

272 Factors affecting the accuracy of NMR chemical shifts

273 Reliably differentiating among different extracted solvation structures requires high accuracy

274 NMR chemical shift predictions. The successful implementation of our framework necessitates

275 adequate consideration of several important factors. First, a key question for the CMD component

276 is the quality of the interatomic potentials since significant deviations in system properties have

277 often been observed compared to experimental data ${ }^{40}$. Second, the DFT level of theory comprising

278 the density functional and basis set is critical for achieving well-converged chemical shieldings.

279 Achieving this convergence for small molecules is relatively straightforward by combining DFT

280 or even coupled cluster calculations with large basis sets. However, this is much more challenging

281 with complexes consisting of multiple species. Therefore, there is a need to balance the cluster size

282 with the quality of the DFT level of theory. In addition, the choice of the implicit solvent model is

283 crucial for approximating the bulk solvent effect. A remarkable number of benchmarking studies

284 have been done on quantum mechanical methods for predicting properties in complex

285 multicomponent battery electrolytes similar to the test case here ${ }^{41-43}$. However, parallel studies for 
286 NMR calculations for these types of systems are still in their infancy. Other factors include 287 selecting an appropriate number of molecules in the chemical reference to account for 288 intermolecular interactions and a representative conformer from each solvation environment. In 289 the following sections, we report the role of each of these factors using results obtained by the 290 framework for the $\mathrm{Mg}(\mathrm{TFSI})_{2} / \mathrm{DME}$ test case system.

\section{$291 \quad$ Role of the force field}

292 The choice of the force field parameters used in CMD simulations can significantly influence 293 the speciation observed in solution, and thus the NMR chemical shift predictions. Therefore, we 294 benchmark the most commonly used and reliable force fields for liquid solutions, including GAFF 295 (FF1), non-polarizable OPLS ${ }^{44}$ (FF2), and polarizable OPLS (FF3) force fields to compare their 296 performance in terms of the solution properties. FF1 and FF2 are computationally less expensive 297 due to their non-polarizable nature and have been used extensively in the battery literature showing 298 satisfactory agreement with experimental findings. FF3, built on top of FF2, allows for a 299 polarizable response of molecules to an electric field using the Drude oscillator model ${ }^{45}$. In this 300 model, particles are added to each polarizable atom to mimic physical dipoles and model the 301 corresponding distortion of electron density.

302 The simulation density $(\rho)$ using the three force fields, shown in Table S2, agrees well with 303 the experimental value. The lowest average error (1.2\%) is achieved with FF3. The RDFs between 304 the cation and oxygen atoms of DME and TFSI- are shown in Fig 2a and 2b, respectively. FF1 305 results in the weakest cation-solvent $\left[\mathrm{Mg}^{2+}\right.$-DME] interaction and the most vital cation-anion $306\left[\mathrm{Mg}^{2+}-\mathrm{TFSI}^{-}\right]$interaction, as evident from the sharp RDF peak between the cation and oxygen 307 atoms of the anion. On the other hand, with FF3, little coordination occurs with the anion (inset of 308 Fig 2b), indicating that solvent molecules dominate the first solvation shell of the cation. Fig 2c 
shows $\mathrm{Mg}^{2+}-\mathrm{O}$ (DME) and $\mathrm{Mg}^{2+}-\mathrm{O}\left(\mathrm{TFSI}^{-}\right)$coordination numbers, calculated by integrating the

310 corresponding RDF curves for the first solvation shell. FF3 results indicate that $\operatorname{Mg}(\mathrm{TFSI})_{2}$ tends

311 to form SSIPs in DME, while FF2 shows that the salt participates in forming CIPs. On the other

312 extreme, FF1 results in the formation of aggregate solvates (AGGs), in which two or more anions

313 coordinate with the cation. An example of the type of coordination represented by the RDFs is

314 displayed in Fig 2d. The tested force fields also result in different percentages of DME and TFSI ${ }^{-}$

315 that coordinate to $\mathrm{Mg}^{2+}$ with two oxygen atoms, i.e., in bidentate configuration, as shown in Fig

316 S5.
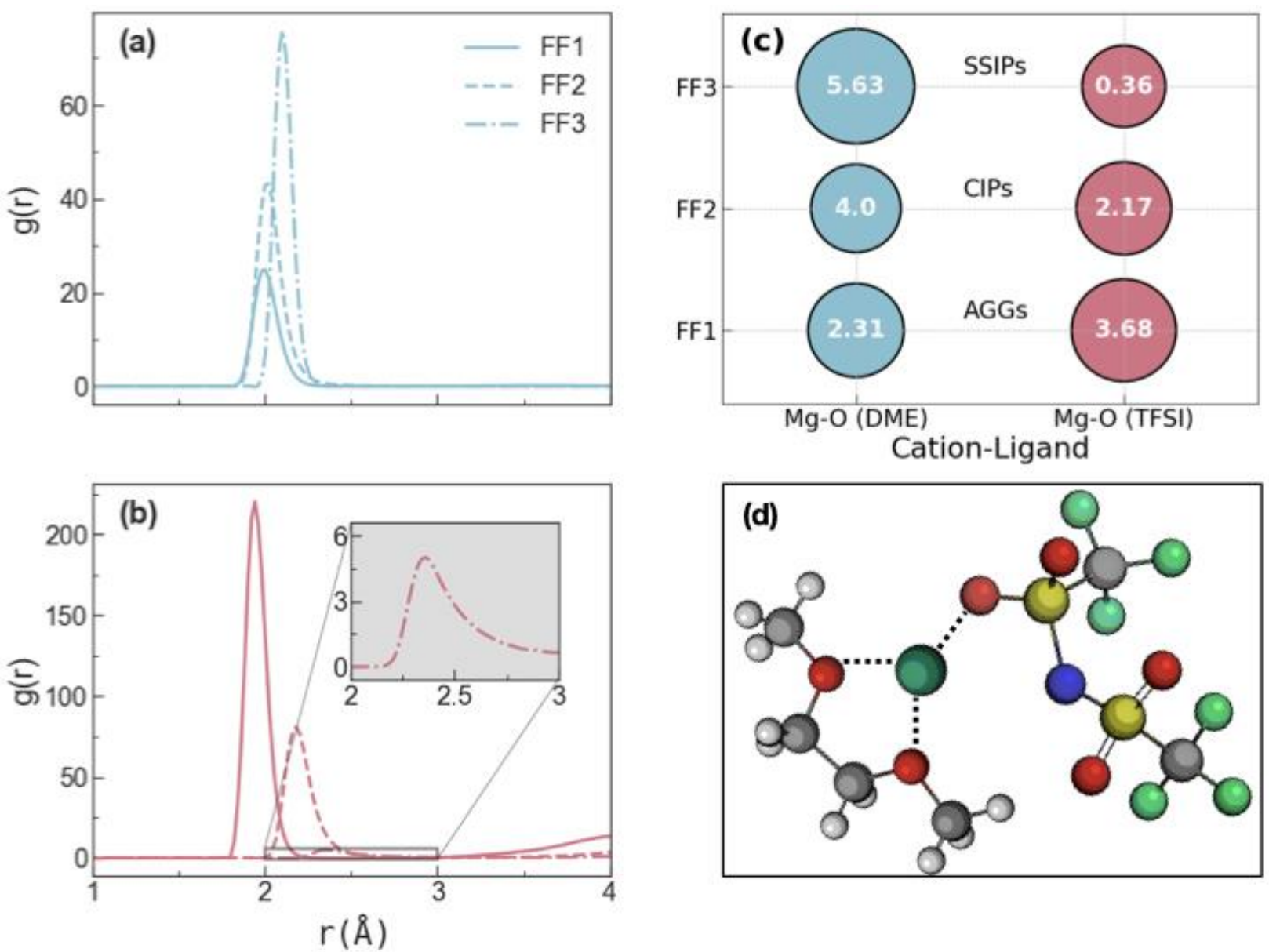

Fig. 2 Structural properties of Mg(TFSI) 2 in DME at 298.15 K using FF1 (GAFF), FF2 (non-polarizable OPLS), and FF3 (polarizable OPLS). RDF of (a) $\mathrm{Mg}^{2+}-\mathrm{O}$ (DME) and (b) $\mathrm{Mg}^{2+}-\mathrm{O}$ (TFSI ${ }^{-}$), (c) coordination numbers with $\mathrm{Mg}^{2+}$ with the corresponding type of structure: solvent separated ion pairs (SSIPs), contact ion pairs (CIPs), and aggregates (AGGs), and (d) corresponding types of coordination with oxygen atoms of DME and TFSI 
The top $\mathrm{Mg}^{2+}$ configurations identified by the framework are provided in Fig S6 and Fig S7.

318 Overall, we find significant differences in the type and distribution of these structures among the

319 tested force fields. For example, the most probable solvation structure predicted with FF1 involves

320 one DME molecule in bidentate configuration and four TFSI ${ }^{-}$anions in monodentate configuration.

321 In addition, rather than forming a single stable solvate like in the case of FF3, the distribution of

322 coordination environments for the cation with FF1 is much more heterogeneous and involves

323 configurations dominated by the anion. With FF2, the electrostatic interaction with the anion is

324 slightly suppressed, and the most probable solvation shell is composed of two DME solvents and

325 TFSI $^{-}$anions participating in bidentate and monodentate configurations, respectively. FF3 results

326 in an $\mathrm{Mg}^{2+}$ solvation shell dominated by three DME molecules participating in bidentate

327 configuration, with only minor structures containing an anion. This configuration has been

328 previously suggested based on experimental measurements of diffusion and Raman and NMR

329 spectroscopy ${ }^{23,26}$, and computationally by Kubisiak and Eilmes ${ }^{46}$ for a concentration range of 0.1 -

$3301 \mathrm{M}$.

331 Variations in the structural properties between the tested force fields are translated to the 332 dynamical behavior of the electrolyte. The distribution of diffusion coefficients (Fig S8) from FF3

333 indicates 2.58 slow DME molecules per $\mathrm{Mg}^{2+}$ cation. This result is in close agreement with the 334 experimentally measured value of $3.0^{23}$ and is consistent with the computed structural properties. 335 FF1 and FF2 predict 1.17 and 1.86 slow DME molecules per $\mathrm{Mg}^{2+}$, respectively. The calculated 336 ionic diffusion coefficients with FF3 are also in better agreement with experimental results (mean 337 absolute error of 20\%), whereas those from FF1 and FF2 are underestimated by approximately $33890 \%$ and $30 \%$, respectively (Fig S9). 
The discrepancies in the predicted properties are not particularly a problem of a specific force

340 field or the $\mathrm{Mg}(\mathrm{TFSI})_{2} / \mathrm{DME}$ system, but rather due to a lack of accounting for the critical

341 interactions in the non-polarizable simulations. The predicted properties using FF3 are the most

342 consistent with previous experimental ${ }^{23,26}$ and computational ${ }^{46}$ studies among the tested force

343 fields. However, the better performance of FF3 comes at the expense of its 2-3 fold higher

344 computational time compared to FF1 and FF2. To summarize, the computational results for the

345 cation-anion motifs and the propensity of the salt to form ion aggregation in the solution are

346 strongly dependent on the type of the force field. Therefore, evaluating the quality of the force

347 field used in the sampling process is a necessary primary step to obtain reliable structures for NMR

348 computations. Here, we proceed with the FF3-predicted solvation structures to report results from

349 the DFT component of the NMR framework.

$350 \quad$ Role of the DFT level of theory

351 We evaluate the performance of selected DFT functionals and basis sets in predicting chemical

352 shifts of ${ }^{25} \mathrm{Mg},{ }^{13} \mathrm{C}$, and ${ }^{1} \mathrm{H}$ of the top configurations and the chemical shifts of ${ }^{13} \mathrm{C}$ and ${ }^{1} \mathrm{H}$

353 resonances in the bulk solution. All calculations presented in this section are performed using the

354 polarizable continuum model (PCM) $)^{47-49}$. The NMR workflow (Fig 1) is designed to be used in

355 high-throughput mode to study speciation evolution in liquid solutions at variable conditions, e.g.,

356 concentration and temperature. Therefore, the comparison is made not only based on accuracy but

357 also on factors that are particularly important for high-throughput simulations (e.g., computational

358 cost and tendency to fail). Predictions from combinations of four commonly utilized DFT

359 functionals (B3LYP ${ }^{50}, \mathrm{M} 06-2 \mathrm{X}^{51}, \mathrm{PBE} \mathrm{PBE}^{52}$, and $\left.\omega \mathrm{B} 97 \mathrm{X}^{39}\right)$ and three Gaussian basis sets (6-

$36031+\mathrm{G}^{*}, 6-311++\mathrm{G}^{* *}$, and def2-TZVP) are compared with experimental NMR data. 
During the benchmark study, the most common failures encountered include failure to 362 converge the geometry to a PES minimum in a finite number of optimization steps, difficulties in 363 converging SCF calculations, and errors in internal coordinate transformations. Around $78 \%$ of 364 the total performed calculations were completed without error correction procedures. Levels of 365 theory primarily involved in the failed calculations include B3LYP/6-31+G* and PBE1PBE 366 hybrid functional coupled with each of the $6-31+\mathrm{G}^{*}$ and $6-311++\mathrm{G}^{* *}$ basis sets. Given that one 367 of our primary goals is to find a level of theory that is not likely to fail with complex 368 multicomponent clusters, these levels of theory are not considered the most appropriate for the 369 required task.

370 The ${ }^{25} \mathrm{Mg}$ NMR results from the top-performing level of theory ( $\omega$ B97X/def2-TZVP) are 371 shown in Fig 3 along with the corresponding structure of the predicted species. A single broad 372 peak is observed, indicating either a single solvation structure or a convolution of multiple 373 structures with a rapid exchange. The predicted ${ }^{25} \mathrm{Mg}$ chemical shift in the most probable 374 configuration is $-0.809 \mathrm{ppm}$, which is highly consistent with the experimental peak center located 375 at $-0.71 \mathrm{ppm}$. Given the broad line width of the ${ }^{25} \mathrm{Mg}$ peak, i.e., the half peak height at 0.83 and $3762.13 \mathrm{ppm}$, the chemical shift of ${ }^{25} \mathrm{Mg}$ in configuration 2 (Table 1) is also deemed to be in 377 satisfactory agreement with experimental data. Therefore, multiple $\mathrm{Mg}^{2+}$ structures that are 378 entirely dissociated from the anion are possible in the solution. Excluding configuration 4, the 379 increase in the ion-dipole interaction between $\mathrm{Mg}^{2+}$ and TFSI - in the following order: configuration $3801<$ configuration $2<$ configuration $3<$ configuration $6<$ configuration 5 leads to the observed 381 monotonic upfield shift in the corresponding ${ }^{25} \mathrm{Mg}$ chemical shift. The presence of loosely packed 382 clusters of $\left[\mathrm{Mg}(\mathrm{DME})_{\mathrm{n}}\right](\mathrm{n} \leq 2)$, i.e., configuration 4 , is attributed to the high degree of freedom 383 and structural flexibility of DME. This type of configuration has been reported to be favorable at 
384 lower concentrations due to lower electrostriction (reduced solvent volume in the $\mathrm{Mg}^{2+}$ solvation 385 shell relative to the bulk) and diminished entropy $\operatorname{loss}^{23}$. On the contrary, higher concentrations $386(0.51 \mathrm{M})$ such as the one used in this study lead to closer distances between $\mathrm{Mg}^{2+}$ ions, resulting 387 in stronger electrostatic interactions and dampened DME motion, thus favoring fully solvated 388 clusters $(\mathrm{n}=3$, configuration 1$)$. This behavior is consistent with the low probability of 389 configuration 4 and the predicted ${ }^{25} \mathrm{Mg}$ chemical shift of this configuration, which is far from the 390 experimental peak center (Fig 3).

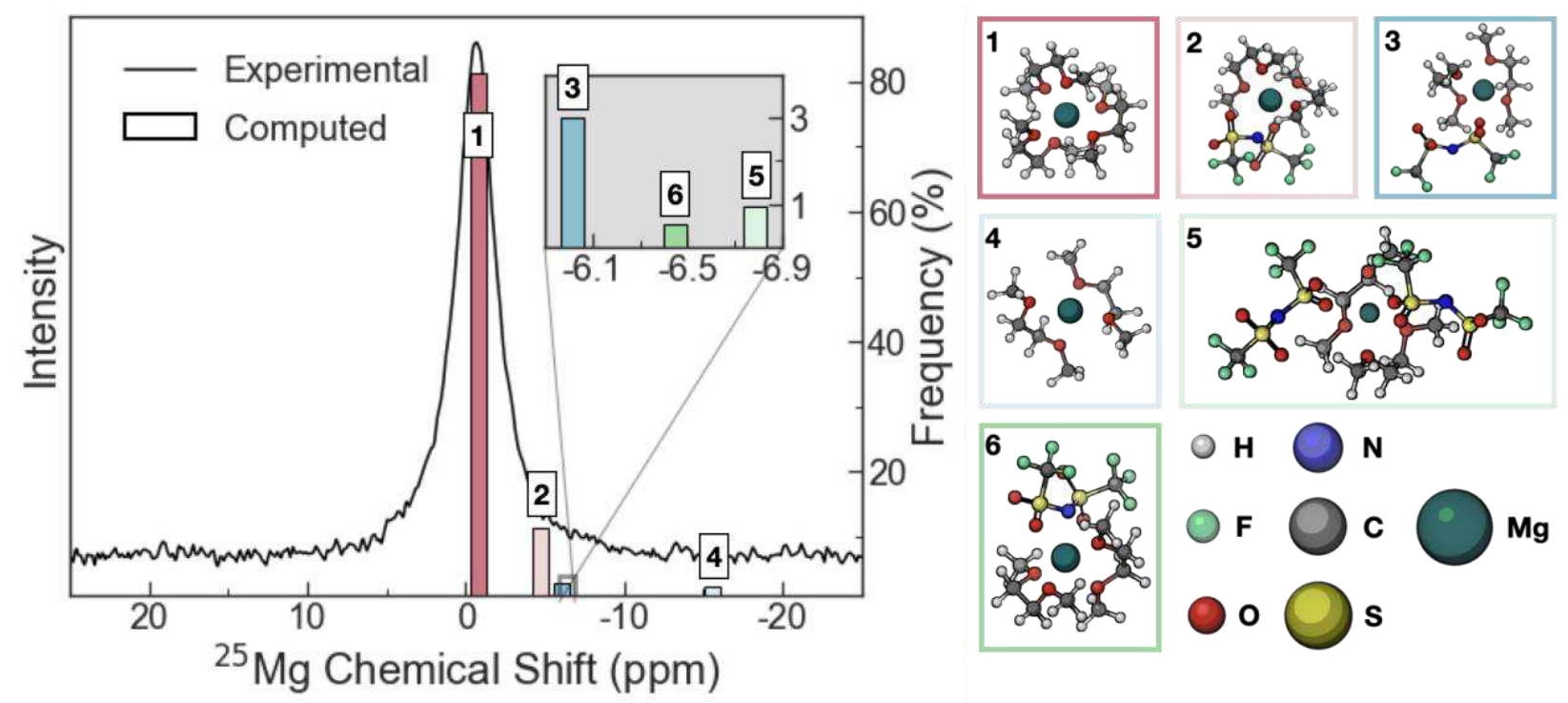

Fig. 3 Predicted ${ }^{25} \mathrm{Mg}$ NMR chemical shifts using the NMR computational protocol and the experimental NMR spectrum along with the corresponding predicted solvation structures of $1: 18 \mathrm{Mg}(\mathrm{TFSI})_{2}$ in DME solution. DFT calculations are performed at the $\omega \mathrm{B} 97 \mathrm{X} / \mathrm{def}$-TZVP level of theory using the PCM solvation model 
Table 1 DFT predicted chemical shifts for 1:18 $\mathrm{Mg}(\mathrm{TFSI})_{2}$ in DME solution along with deviations from experimental data

\begin{tabular}{|c|c|c|c|c|c|c|}
\hline \multirow[t]{2}{*}{ Molecule $^{\mathrm{a}}$} & \multicolumn{2}{|c|}{$\delta{ }^{25} \mathrm{Mg}(\mathrm{ppm})$} & \multicolumn{2}{|c|}{$\delta{ }^{13} \mathrm{C}(\mathrm{ppm})$} & \multicolumn{2}{|c|}{$\delta{ }^{1} \mathrm{H}(\mathrm{ppm})$} \\
\hline & $\mathrm{PCM}^{\mathrm{b}, \mathrm{c}}$ & $\mathrm{SMD}^{\mathrm{b}, \mathrm{c}}$ & Shift $\mathrm{t}^{\mathrm{c}, \mathrm{d}}$ & Deviation $^{\mathrm{e}}$ & Shift ${ }^{\mathrm{d}, \mathrm{f}}$ & Deviation $^{\mathrm{e}}$ \\
\hline Bulk DME & & & $\mathrm{CH}_{2}: 72.32$ & $\mathrm{CH}_{2}: 0.83$ & $\mathrm{CH}_{2}: 3.74$ & $\mathrm{CH}_{2}: 0.06$ \\
\hline & & & $\mathrm{CH}_{3}: 59.16$ & $\mathrm{CH}_{3}: 0.27$ & $\mathrm{CH}_{3}: 3.64$ & $\mathrm{CH}_{3}:-0.01$ \\
\hline Configuration 1 & -0.809 & 0.283 & $\mathrm{CH}_{2}: 72.62$ & $\mathrm{CH}_{2}:-0.26$ & $\mathrm{CH}_{2}: 4.24$ & $\mathrm{CH}_{2}: 0.16$ \\
\hline$\left[\mathrm{Mg}(\mathrm{DME})_{3}\right]^{2+}$ & & & $\mathrm{CH}_{3}: 62.70$ & $\mathrm{CH}_{3}:-0.09$ & $\mathrm{CH}_{3}: 4.07$ & $\mathrm{CH}_{3}: 0.29$ \\
\hline Configuration 2 & -4.741 & -4.649 & $\mathrm{CH}_{2}: 72.50$ & $\mathrm{CH}_{2}:-0.14$ & $\mathrm{CH}_{2}: 4.20$ & $\mathrm{CH}_{2}: 0.20$ \\
\hline $\left.\operatorname{Mg}(\mathrm{DME})_{2}(\mathrm{TFSI})\right]^{+}$ & & & $\mathrm{CH}_{3}: 62.18$ & $\mathrm{CH}_{3}: 0.43$ & $\mathrm{CH}_{3}: 4.11$ & $\mathrm{CH}_{3}:-0.01$ \\
\hline Configuration 3 & -6.016 & 2.784 & $\mathrm{CH}_{2}: 72.16$ & $\mathrm{CH}_{2}: 0.20$ & $\mathrm{CH}_{2}: 4.27$ & $\mathrm{CH}_{2}: 0.13$ \\
\hline $\left.\operatorname{Mg}(\mathrm{DME})_{2}(\mathrm{TFSI})\right]^{+}$ & & & $\mathrm{CH}_{3}: 61.64$ & $\mathrm{CH}_{3}: 0.97$ & $\mathrm{CH}_{3}: 4.15$ & $\mathrm{CH}_{3}:-0.05$ \\
\hline Configuration 4 & -15.559 & -3.982 & $\mathrm{CH}_{2}: 73.37$ & $\mathrm{CH}_{2}:-1.01$ & $\mathrm{CH}_{2}: 4.32$ & $\mathrm{CH}_{2}: 0.08$ \\
\hline$\left[\mathrm{Mg}(\mathrm{DME})_{2}\right]^{2+}$ & & & $\mathrm{CH}_{3}: 62.73$ & $\mathrm{CH}_{3}:-0.12$ & $\mathrm{CH}_{3}: 4.14$ & $\mathrm{CH}_{3}:-0.04$ \\
\hline Configuration 5 & -6.785 & -6.196 & $\mathrm{CH}_{2}: 71.85$ & $\mathrm{CH}_{2}: 0.51$ & $\mathrm{CH}_{2}: 4.10$ & $\mathrm{CH}_{2}: 0.30$ \\
\hline$\left[\mathrm{Mg}(\mathrm{DME})_{2}(\mathrm{TFSI})_{2}\right]$ & & & $\mathrm{CH}_{3}: 62.57$ & $\mathrm{CH}_{3}: 0.04$ & $\mathrm{CH}_{3}: 4.14$ & $\mathrm{CH}_{3}:-0.04$ \\
\hline Configuration 6 & -6.450 & -5.815 & $\mathrm{CH}_{2}: 72.68$ & $\mathrm{CH}_{2}:-0.32$ & $\mathrm{CH}_{2}: 4.29$ & $\mathrm{CH}_{2}: 0.11$ \\
\hline$\left[\mathrm{Mg}(\mathrm{DME})_{2}(\mathrm{TFSI})\right]^{+}$ & & & $\mathrm{CH}_{3}: 62.52$ & $\mathrm{CH}_{3}: 0.09$ & $\mathrm{CH}_{3}: 4.13$ & $\mathrm{CH}_{3}:-0.03$ \\
\hline
\end{tabular}

${ }^{\text {a }}$ For the difference between configurations 2, 3, and 6, refer to Fig S3,

${ }^{\mathrm{b}}$ Compared to experimental peak center at $-0.71 \mathrm{ppm}$,

c Using $\omega B$ 97X/def2-TZVP,

d Using PCM model,

${ }^{\mathrm{e}}$ Deviation $=\delta_{\text {exp }}-\delta_{D F T}$,

${ }^{\mathrm{f}}$ Using M06-2X/def2-TZVP. 
The benchmarking results for ${ }^{25} \mathrm{Mg}$ chemical shift calculations are displayed in Fig S10. On

392 average, going from left to right, i.e., increasing the number of basis functions, moves most of the

393 predicted chemical shifts corresponding to different electronic environments surrounding the ${ }^{25} \mathrm{Mg}$

394 nucleus within the bounds of the observed NMR spectrum. From top to bottom, significant

395 variations are observed in the predicted chemical shifts using the four functionals with $6-31+\mathrm{G}^{*}$,

396 while this difference is less clear with def2-TZVP. In addition, we find that different levels of

397 theory can lead to contradictory conclusions regarding the dominant species in solution. For

398 example, the structure is predicted to be $\left[\mathrm{Mg}(\mathrm{DME})_{2}(\mathrm{TFSI})\right]^{+}$using PBE1PBE/6-31+G* while the

399 fully solvated $\left[\mathrm{Mg}(\mathrm{DME})_{3}\right]^{2+}$ is found with PBE1PBE/def2-TZVP.

$400 \quad$ Fig 4 shows ${ }^{13} \mathrm{C}$ NMR shifts assigned to $\mathrm{CH}_{3}$ of $\mathrm{DME}$ existing in the bulk solution (labeled

401 'free $\mathrm{CH}_{3}$ ') and DME coordinated to $\mathrm{Mg}^{2+}$ (labeled 'bound $\mathrm{CH}_{3}$ ') from DFT predictions and

402 experimental measurements. Similar plots for ${ }^{13} \mathrm{C}$ shifts assigned to $\mathrm{CH}_{2}$ and ${ }^{1} \mathrm{H}$ shifts assigned to

$403 \mathrm{CH}_{3}$ and $\mathrm{CH}_{2}$ of both types of DME molecules are shown in Figs S11-S13, respectively. While

404 free and bound DME molecules are distinguishable from experimental and predicted ${ }^{13} \mathrm{C}$ and ${ }^{1} \mathrm{H}$

405 NMR chemical shifts, it is impossible to differentiate between bound DME at different

406 configurations identified in this work. The spectroscopic differences between the structures may

407 be subtle (see, for example, Table 1 for ${ }^{13} \mathrm{C}$ and ${ }^{1} \mathrm{H}$ chemical shifts in different configurations). On

408 the contrary, ${ }^{25} \mathrm{Mg}$ chemical shifts can be utilized for this purpose, whereby changes in charge

409 density localization on different $\mathrm{Mg}^{2+}$ complexes directly alter the screening effects experienced

410 by the ${ }^{25} \mathrm{Mg}$ nucleus, thus giving rise to different NMR responses. As displayed in Fig 4 and Fig

$411 \mathrm{~S} 11$, the highest deviation from experimental ${ }^{13} \mathrm{C}$ shifts are obtained with the 6-31+G* and 6-

$412311++\mathrm{G}^{* *}$ basis sets combined with any tested density functional. The basis set from the 'def2'

413 family of Alrichs and coworkers ${ }^{53}$, particularly in combination with $\omega \mathrm{B} 97 \mathrm{X}$, leads to ${ }^{13} \mathrm{C}$ NMR 
414 chemical shift error that approaches the underlying uncertainty in experimental measurements

415 (Table 1). Fig S12 and S13 indicate that for ${ }^{1} \mathrm{H}$ chemical shifts, M06-2X/def2-TZVP outperforms

416 the other tested levels of theory with absolute errors between 0.01 and $0.3 \mathrm{ppm}$ (Table 1).

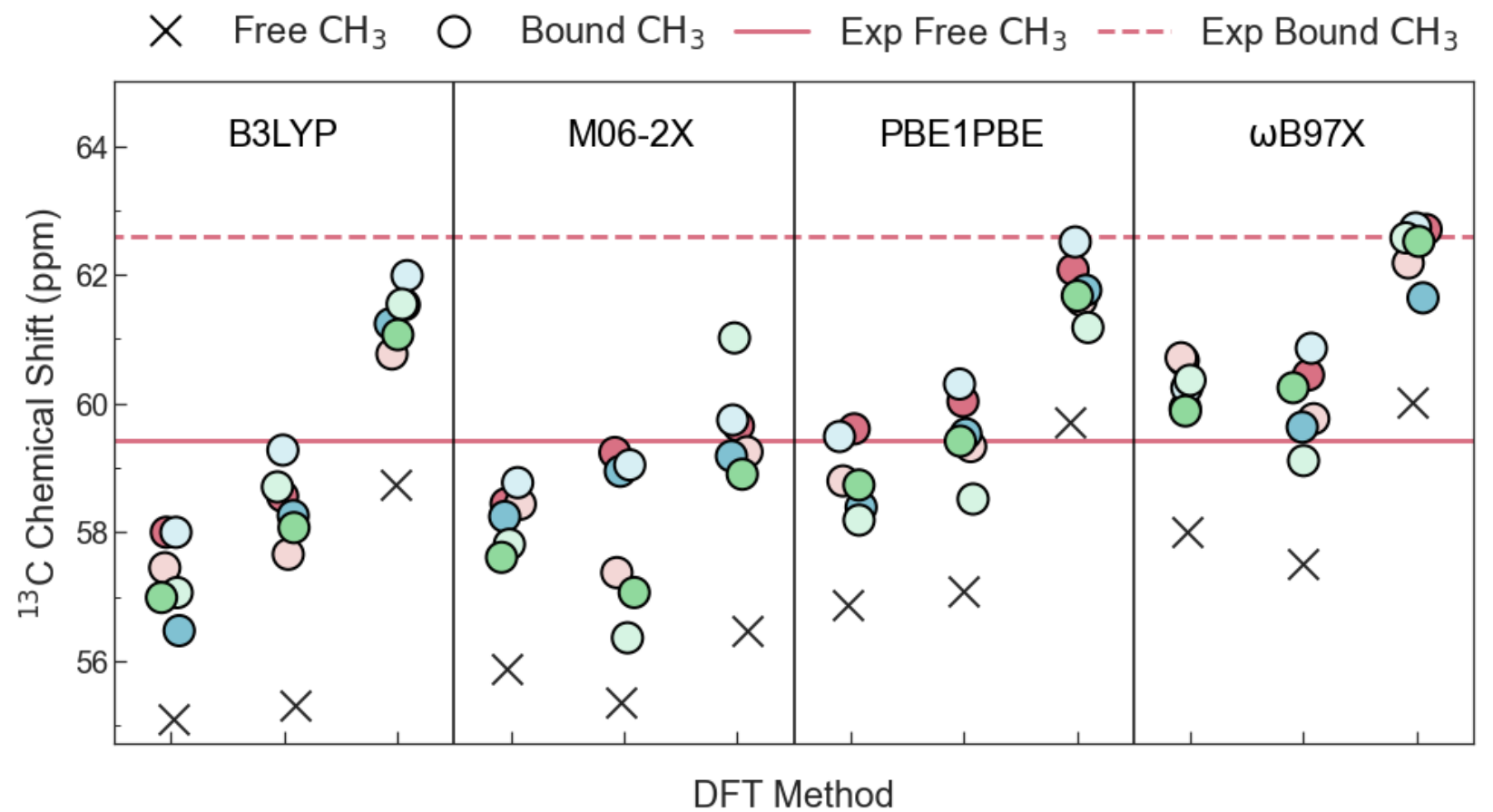

Fig. 4 Strip plot of the computed and experimental ${ }^{13} \mathrm{C}$ NMR chemical shifts assigned to $\mathrm{CH}_{3}$ of $\mathrm{DME}$ coordinated to $\mathrm{Mg}^{2+}$ (labeled Bound $\mathrm{CH}_{3}$ ) and $\mathrm{CH}_{3}$ of free DME (labeled Free $\mathrm{CH}_{3}$ ). For color code of 'Bound $\mathrm{CH}_{3}$ ', please refer to Fig 3. Results from each DFT functional are shown with the basis sets in the following order: 6-31+G*, 6-311++ $\mathrm{G}^{* *}$, and def2-TZVP

417 We conclude that the choice of the basis set has the highest impact on the accuracy of NMR

418 chemical shift predictions. The $6-31+G^{*}$ basis set is ruled out as a suitable basis set for NMR

419 calculations of complexes similar to those studied herein due to its degraded accuracy compared

420 to other basis sets, despite its lower computational cost (see Fig S14 for timings). For ${ }^{25} \mathrm{Mg}$ and

$421{ }^{13} \mathrm{C}$ chemical shifts, the $\omega \mathrm{B} 97 \mathrm{X} / \mathrm{def} 2$-TZVP level of theory is recommended if computational

422 resources are available as its remarkable accuracy and the applicability of def2-TZVP to broader

423 chemical systems make it well worth the additional cost. If computational resources are limited,

$424 \mathrm{M} 06-2 \mathrm{X} / 6-311++\mathrm{G}^{* *}$ is recommended for ${ }^{25} \mathrm{Mg}$ shifts as its cost is not prohibitive while still 
425 predicting the correct $\mathrm{Mg}^{2+}$ solvation structure. Finally, M06-2X with def2-TZVP or 6-311++G**

426 are recommended for ${ }^{1} \mathrm{H}$ chemical shift predictions.

\section{Effect of geometry optimization}

428 To examine the possibility of making DFT calculations more affordable, we calculated the

$429{ }^{25} \mathrm{Mg}$ chemical shift of 33 pre-relaxed clusters extracted from CMD simulations. We then compare

430 their deviation from calculations utilizing optimized geometries at the same level of theory (Fig

431 S15). We find a mean absolute deviation of $\sim 37.6 \mathrm{ppm}$ between the two types of calculations,

432 with a systematic downfield shift from calculations utilizing fully optimized structures. This result

433 is not surprising due to the sensitivity of the ${ }^{25} \mathrm{Mg}$ nucleus to subtle differences in the local structure

434 and coordination environment. Therefore, relaxing the structures ensures that 'reasonable

435 geometries' are used, and therefore is a prerequisite for obtaining accurate NMR chemical shifts

436 that are comparable to experimental measurements.

437 Choice of the chemical reference

438 Because water is selected as the ${ }^{1} \mathrm{H}$ chemical shift reference, another consideration is the 439 accurate computational representation of the effect of strong hydrogen bonding among water 440 molecules. To this end, calculations on clusters of $\left(\mathrm{H}_{2} \mathrm{O}\right)_{n}(\mathrm{n}=1-4,6,8,10,12)$ are performed.

441 At the M06-2X/def2-TZVP level of theory, the isotropic shielding constant of ${ }^{1} \mathrm{H}$ moves upfield 442 when the number of water molecules increases and tends to converge at $\sim 27.6 \mathrm{ppm}$ for eight water 443 molecules. In addition, multiple clusters for non-hydrogen-bonding dimethylsulfoxide (DMSO)n $444(n=1-4,6)$, used as a reference in ${ }^{13} \mathrm{C}$ chemical shift calculations, are considered. The use of a 445 DMSO dimer is found to be sufficient, whereby the ${ }^{13} \mathrm{C}$ isotropic shielding constant converges at $446 \sim 150 \mathrm{ppm}$ at the $\omega \mathrm{B} 97 \mathrm{X} / \mathrm{def} 2-\mathrm{TZVP}$ level of theory. Finally, since intermolecular interactions 447 inevitably exist in DME solution, calculations on $(\mathrm{DME}) \mathrm{n}_{\mathrm{n}}(\mathrm{n}=1-4)$ are carried out for predicting 
448 the ${ }^{13} \mathrm{C}$ and ${ }^{1} \mathrm{H}$ chemical shifts of DME molecules in the bulk solution, and (DME) 2 is found to

449 result in bulk $\mathrm{CH}_{3}$ and $\mathrm{CH}_{2}$ chemical shifts that reproduce the experimental data. All calculated

450 isotropic shielding constants for $\mathrm{H}_{2} \mathrm{O}$, DMSO, and DME clusters are included in the dataset

451 associated with this work.

452 Role of the implicit solvation model

453 In addition to the explicit solvent molecules modeled in the $\mathrm{Mg}^{2+}$ first solvation shell, an 454 implicit model is used to incorporate long-range electrostatic effects. Implicit solvent models have 455 the advantage of reducing the number of degrees of freedom of the environment (solvent), thereby 456 decreasing the computational cost to describe the dielectric continuum outside the solute cavity. 457 SMD is reliable in many applications ${ }^{54}$ and therefore is compared to the PCM results in this work. 458 As evident from the data in Table 1, both methods lead to similar interpretations of experimental 459 results in terms of the most probable solvation structure. However, the PCM method predicts more 460 accurately the chemical shift of the top configuration. The only significant differences between the 461 two methods are for configurations 3 and 4. Similarly, more minor errors associated with ${ }^{13} \mathrm{C}$ and

$462{ }^{1} \mathrm{H}$ chemical shifts are achieved with PCM than the SMD model using the $\omega$ B97X/def2-TZVP and 463 M06-2X/def2-TZVP levels of theory, respectively (Fig S16).

\section{$464 \quad \underline{\text { Role of conformer }}$}

465 Another consideration in the NMR framework is that it utilizes the lowest-energy conformer 466 from each configuration to initialize the NMR DFT calculations. Previous NMR DFT studies have 467 also reported findings on possible solvation structures based on a single conformer ${ }^{20,24,55}$. 468 However, the measured shift is the weighted average of chemical shifts of all possible conformers 469 in solution during the NMR acquisition time. Therefore, we assess the sensitivity of DFT chemical 470 shifts to conformer sampling by starting from the MMFF94 energies of a total of $\sim 270,000$ 
471 conformers of configuration 1 and $~ 4,000$ conformers of configuration 2 extracted from CMD

472 simulations. From each configuration, 15 conformers spanning the entire energy range are selected

473 to initialize full NMR calculations that include geometry optimization, frequency, and chemical

474 shift estimation at the $\omega B$ 97X/def2-TZVP level of theory using the PCM solvation model.

475 Boltzmann averaging is done according to the equation shown in Fig 5 to calculate the ensemble

476 NMR chemical shift. Because optimization at the higher level of theory leads to the reordering of

477 conformational energies, the results are reported relative to the MM global minimum energy

478 conformer. The plots in Fig 5 show the mean difference (including the $95 \%$ confidence interval)

479 between the Boltzmann average NMR chemical shift for the entire ensemble, $\langle\delta\rangle$, and our initial

480 chemical shift estimation, $\delta_{0}$, as a function of the number of optimizations performed. We note

481 that for each number of optimized structures $\left(N_{\text {opt }}\right)$ shown on the X-axis of Fig 5 , the calculated

$482\langle\delta\rangle$ is the result of averaging over all possible combinations of $N_{\text {opt }}$ from a pool of 15 structures,

483 with a restriction that the MM global minimum energy conformer is included in these

484 combinations. To maintain statistical significance, only $N_{o p t}$ resulting in more than 30 possible

485 combinations are used, thus $N_{\text {opt }}=2,13,14,15$ are excluded from the analysis. Variable degrees

486 of errors are obtained with each nucleus type, with the highest difference in the ${ }^{25} \mathrm{Mg}$ chemical

487 shift. In this electrolyte system, a maximum unsigned error of $1.2 \mathrm{ppm}$ in the ${ }^{25} \mathrm{Mg}$ chemical shift

488 of configuration 1 upon excluding conformational sampling does not alter the interpretation of

489 experimental findings in terms of the most probable solvation structure while saving $15 \times$ the

490 computational resources. Nevertheless, conformational sampling has a more pronounced impact

491 on other less probable solvation structures like configuration 2, for which an error of $4.4 \mathrm{ppm}$ is

492 incurred if only the MM global minimum is considered for calculations at the higher level of

493 theory. Significantly lower errors are obtained for ${ }^{13} \mathrm{C}$ chemical shifts of $\mathrm{CH}_{2}$ and $\mathrm{CH}_{3}$ groups, 
494 while ${ }^{1} \mathrm{H}$ chemical shifts are insensitive to conformer sampling regardless of the type of 495 configuration to which the proton belongs. Therefore, an evaluation of the impact of 496 conformational sampling on DFT predictions for a nucleus of interest should be done whenever 497 possible to boost the confidence in correlations established between experiments and the results of 498 the computational framework described in this work. Such a process would determine whether the 499 conformer issue is critical in the examined case study to possibly avoid instances of multiple 500 conformers that would need to be considered.
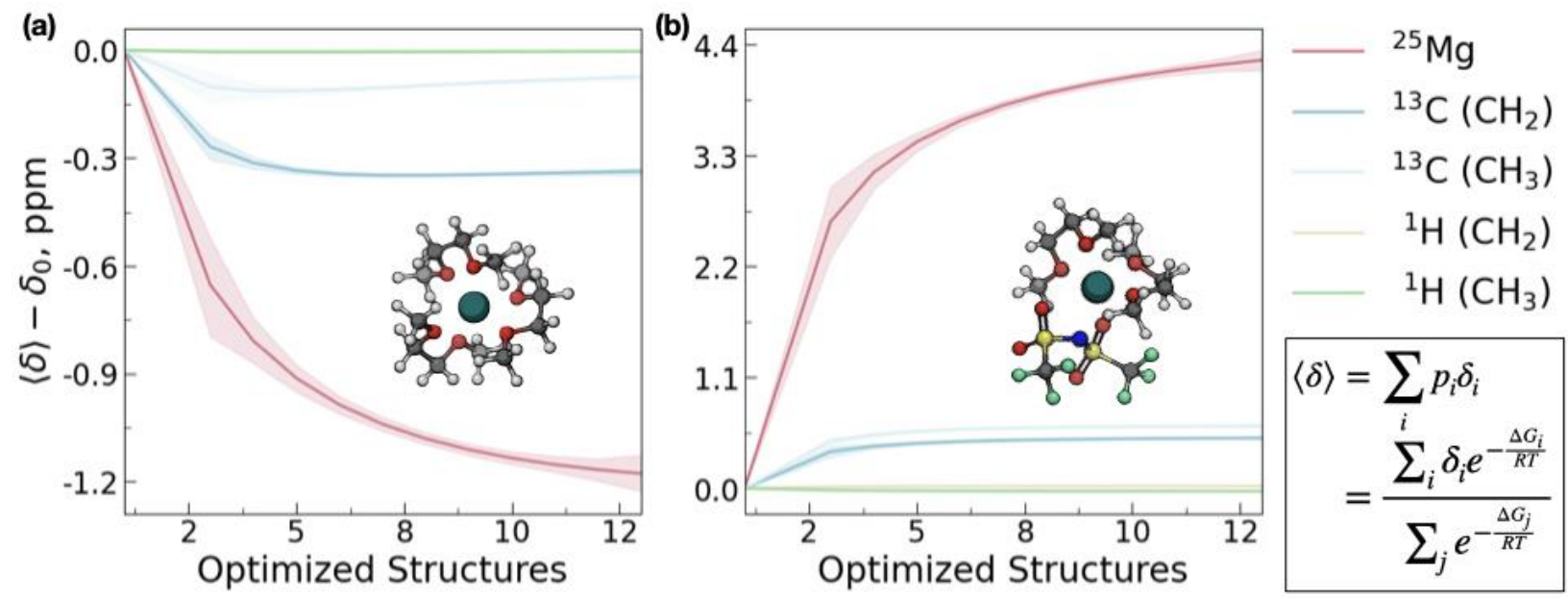

Fig. 5 Effect of multiple conformers for (a) configuration 1 and (b) configuration 2 on ${ }^{25} \mathrm{Mg},{ }^{13} \mathrm{C}$, and ${ }^{1} \mathrm{H}$ NMR chemical shifts. In the equation of the Boltzmann weighted average of the chemical shift $\langle\delta\rangle, p_{i}$ and $\Delta G_{i}$ are the Boltzmann weight and the formation energy of structure $i$ relative to the most stable configuration predicted by DFT, respectively

CONCLUSIONS

In conclusion, we have developed and tested a computational framework that couples firstprinciple calculations with CMD simulations to robustly and efficiently calculate, analyze, and

504 store NMR chemical shifts from a variety of molecules in liquid solutions. The framework overcomes limitations in current NMR computational studies such as the Edisonian approach in selecting possible solvation structures and the significant time required for manual file 
508 to accurately identify multiple stable species present in the solution that contribute to the overall

509 NMR spectral shape. Minimal inputs comprising structures of species in solution and their force

510 field parameters are required to obtain accurate shifts, but the calculation procedure can be tuned

511 by overriding default inputs like the level of theory and solvation model. Factors such as the choice

512 of the force field used to identify the type of speciation in solution, DFT level of theory, implicit

513 solvation model, and conformer sampling are critical in determining the accuracy of predictions

514 made by the framework. We have successfully applied the framework to calculate chemical shifts

515 in a complex multicomponent $\operatorname{Mg}(\mathrm{TFSI})_{2} / \mathrm{DME}$ solution and resolved the discrepancy in the

516 literature regarding the $\mathrm{Mg}^{2+}$ solvation structure in this solution. Our results show formation of

517 solvent separated ion pairs in this electrolyte which is consistent with the experimental NMR

518 results reported in this work and the previously reported SCXRD results ${ }^{26}$. The benchmark test

519 case shows that our procedure can generate reliable results that can facilitate NMR deconvolution

520 assignments to determine ionic association interactions within liquid solutions similar to those

521 reported in this work. An extension of this framework is under development and will be

522 successfully added to the existing one. Features that will be supported include the ability to explore

523 the role of the second solvation shell and coupling this strategy with a more detailed analysis of

524 the exchange dynamics in the solution. In addition, support for performing automated polarizable

525 CMD simulations using the thermalized Drude dipole method as implemented in LAMMPS will

526 be added. The current and extended framework will be used to study other monovalent and

527 multivalent electrolytes whose structure is not intuitive or when the chemical and parameter spaces

528 are too large for human search using conventional non-automated methods. Data collected from

529 the framework is expected to provide fingerprints to guide future experimental investigations of

530 liquid solutions with optimal properties. 


\section{METHODS}

\section{Computational}

533 CMD simulations are performed using the LAMMPS simulation package ${ }^{34}$ version 3Mar2020

534 (http://lammps.sandia.gov). Initial configurations of ions in the solvent are first obtained by

535 randomly packing the molecules in a cubic box of size $5 \times 5 \times 5 \mathrm{~nm}^{3}$ with periodicity in $\mathrm{XYZ}$

536 directions using the PACKMOL package ${ }^{56}$. We consider MgTFSI 2 in DME at a salt-to-solvent

537 ratio of 1:18. In FF1, i.e., $\mathrm{GAFF}^{36}$ parameterization, $\mathrm{TFSI}^{-}$and DME parameters are obtained by

538 first generating the electrostatic potential of single molecules in Gaussian $16^{33}$ at the B3LYP/6-

$53931+\mathrm{G}^{*}$ level of theory and fitting the electrostatic potential surface of the optimized structures

540 using the RESP method in Antechamber ${ }^{57}$. AMBER force field parameters by Aqvist are used for

$541 \mathrm{Mg}$ cations ${ }^{58}$. FF2, corresponding to the $\mathrm{OPLS}^{36}$ force field, uses TFSI bonded parameters by

542 Lopes/Pádua ${ }^{59}$ and nonbonded parameters by Köddermann ${ }^{60}$. DME parameters are taken from the

543 work of Anderson and Wilson ${ }^{61}$ except for the parameters of C-C-O-C and O-C-C-O dihedrals,

544 which are based on GAFF parameterization ${ }^{36}$. Lastly, based on FF2, we build FF3

545 parameterization that includes polarization effects via the classical Drude oscillators mode ${ }^{45,62}$.

546 Drude particles are attached to all atoms, excluding hydrogen and $\mathrm{Mg}^{2+}$ due to their relatively small

547 polarizabilities. Atomic polarizabilities and charges for TFSI are based on the APPLE\&P force

548 field $^{63}$, whereas those for DME are taken from work on poly(ethylene oxide) ${ }^{64}$. Nonbonded

549 parameters for $\mathrm{Mg}^{2+}$ cations are adapted from AMOEBA-PRO-13-FF ${ }^{65}$. Force field parameters

550 used in this work are listed in Tables S3-S7.

551 Lennard Jones interactions are truncated at a cutoff distance of $1.2 \mathrm{~nm}$, and the particle-particle

552 particle-mesh (PPPM) ${ }^{66}$ method is used to handle long-range electrostatic interactions. With FF3,

553 a Thole damping factor ${ }^{67}$ of 1.0 is used to smear the neighboring induced dipoles located on the 
554 same molecule and prevent the 'polarization catastrophe ${ }^{168}$. Initial structures are subjected to a two-

555 step energy minimization, first using the steepest descent algorithm employing convergence

556 criteria of $1,000 \mathrm{kcal} / \mathrm{mol} \AA$ and then using a conjugated-gradient minimization scheme with an 557 energy convergence criteria of $10 \mathrm{kcal} / \mathrm{mol} \AA$. The two-step minimization allows for the release of 558 strained contacts in the initial configuration. Isothermal-isobaric simulations (NPT) are performed 559 to obtain the correct density on the minimized system using a Nosé/Hoover temperature thermostat 560 and pressure barostat to maintain the temperature at $298.15 \mathrm{~K}$ and the pressure at 1 atm for $2 \mathrm{~ns}$.

561 With FF3, Drude particles are thermalized at a lower temperature relative to Drude cores to avoid 562 fast vibrations of the small reduced masses, thus allowing the use of a reasonable time step. The 563 system is then melted to $500.15 \mathrm{~K}$ for $2 \mathrm{~ns}$ and subsequently quenched to $298.15 \mathrm{~K}$ for $3 \mathrm{~ns}$. 564 Following that, canonical ensemble (NVT) simulations are performed for $50 \mathrm{~ns}$ using a time step 565 of $0.001 \mathrm{ps}$ at $298.15 \mathrm{~K}$ to equilibrate the system. Molecular trajectories are sampled every $5 \mathrm{ps,}$ 566 resulting in 10,000 snapshots, from which properties of interest are calculated.

567 All DFT calculations are performed using Gaussian $16^{33}$. Magnetic shieldings are calculated 568 for the extracted $\mathrm{Mg}^{2+}$ clusters, ranging in size from 33 to 78 atoms. The benchmark study is 569 performed with twelve combinations of functionals and basis sets (Fig 4) chosen due to their broad 570 application in the NMR literature. An ultrafine integration grid is employed, and van der Waals 571 interactions are treated using Grimme dispersion correction (D3) ${ }^{69}$ with the B3LYP, M06-2X, and 572 PBE1PBE functionals. Besides the explicit solvent model used in this work, bulk solvent effects 573 are described using a continuum model, particularly $\mathrm{PCM}^{47-49}$ or $\mathrm{SMD}^{54}$. Following the 574 optimization and frequency steps, magnetic response calculations are performed using the gauge575 independent atomic orbital (GIAO) $)^{70,71}$ method at the same level of theory. Chemical shifts are 576 converted to the experimentally observed scale using $\delta_{\text {cluster }}=\sigma_{\text {ref }}-\sigma_{\text {cluster }}$, where $\delta_{\text {cluster }}$ 
577 and $\sigma_{\text {cluster }}$ are the chemical shift and the isotropic shielding constant of the nucleus of interest in

578 a given cluster, respectively, and $\sigma_{\text {ref }}$ is the calculated isotropic shielding constant of the same

579 nucleus in a suitable reference compound. We use an $\mathrm{Mg}^{2+}$ ion coordinated octahedrally by six

580 water molecules, dimethyl sulfoxide, and water, as the chemical references for ${ }^{25} \mathrm{Mg},{ }^{13} \mathrm{C}$, and ${ }^{1} \mathrm{H}$,

581 respectively. To reduce systematic errors, we use secondary references (TMS) by adding 39.5 and

$5824.7 \mathrm{ppm}$ to the calculated chemical shifts of carbon and proton, respectively. These values

583 correspond to the experimental chemical shifts of the secondary references relative to the primary

584 standards. We again stress that all the steps described here are automated within our computational

585 framework except for the polarizable CMD simulations.

\section{Experimental}

$587 \mathrm{Mg}(\mathrm{TFSI})_{2}\left(99.5 \%\right.$, Solvionic) were dried for 48 hours under vacuum at $180{ }^{\circ} \mathrm{C}$, and the $\mathrm{DME}$ 588 solvent (Battery-grade, Gotion) was further dried over activated $3 \AA$ molecular sieves in a 589 glovebox until its water content was determined to be below $10 \mathrm{ppm}$ using a Karl-Fisher Titrator 590 (Metrohm). $\mathrm{Mg}$ (TFSI) $2 / \mathrm{DME}$ solutions were prepared inside a glovebox filled with nitrogen right 591 before NMR measurements. ${ }^{1} \mathrm{H}$ and ${ }^{13} \mathrm{C}$ NMR measurements were performed on a Varian DDRS 592 spectrometer with a $17.6 \mathrm{~T}$ magnet using a broad-band (BBO) probe with ${ }^{1} \mathrm{H}$ and ${ }^{13} \mathrm{C}$ Larmor 593 frequencies of 748.1 and $188.1 \mathrm{MHz}$, respectively. The $90^{\circ}$ pulse widths were $16 \mu$ s for ${ }^{1} \mathrm{H}$ and 16 $594 \mu$ s for ${ }^{13} \mathrm{C} .{ }^{1} \mathrm{H}$ spectra were collected using $30^{\circ}$ pulses with a transition number of 16 and a recycle 595 delay of $20 \mathrm{~s}$ with a coaxial tube holding $\mathrm{Mg}(\mathrm{TFSI})_{2} / \mathrm{DME}$ solution and an outer NMR tube holding $596 \mathrm{D}_{2} \mathrm{O}\left(99.9 \%\right.$, from Sigma Aldrich) as an external reference at $4.77 \mathrm{ppm} .{ }^{13} \mathrm{C}$ spectra were collected 597 using $30^{\circ}$ pulses with averaging of 1024 transients and a recycle delay of $12 \mathrm{~s}$ using a thin-wall 5 $598 \mathrm{~mm}$ NMR tube. ${ }^{25} \mathrm{Mg}$ NMR spectra were collected at a $14.1 \mathrm{~T}$ magnet (Varian DDR spectrometer) 599 with a ${ }^{25} \mathrm{Mg}$ Larmor frequency of $36.7 \mathrm{MHz}$ and a $90^{\circ}$ pulse width of $20 \mu$ s. A small tip angle of 
$60015^{\circ}$ with a recycle delay of $0.1 \mathrm{~s}$ was used and 128,000 transients were acquired. In order to

601 minimize the spectrometer drift effect on chemical shift, DMSO-d6 and $5 \mathrm{M} \mathrm{MgCl}_{2}$ were used to

602 reference ${ }^{13} \mathrm{C}(39.52 \mathrm{ppm})$ and ${ }^{25} \mathrm{Mg}(0 \mathrm{ppm})$, respectively, right before each NMR measurement.

\section{DATA AVAILABILITY}

604 The dataset used to generate the results in this work along with the optimized 3D structures in

$605 \mathrm{XYZ}$ format are available in the repository at https://github.com/rashatwi/nmr-dataset.

606 CODE AVAILABILITY

607 The open-source LAMMPS-code is used in the CMD simulations while the proprietary Gaussian-

608 code is primarily used in the DFT calculations. The framework shown in Fig 1 is implemented

609 using the MISPR infrastructure, which defines, executes, manages, and stores DFT and CMD

610 workflows. The codes used in this work will be made publicly available with the future release of

611 the MISPR package.

\section{ACKNOWLEDGMENTS}

613 The authors thank Xiaohui Qu (Brookhaven National Laboratory) for the helpful discussions. High

614 performance computational resources for this research were provided by the Extreme Science and

615 Engineering Discovery Environment (XSEDE) Bridges and Comet computational resources

616 (Charge number: TG-DMR 190087), which is supported by National Science Foundation (NSF)

617 grant number ACI-1548562. This work also used computational resources at the Stony Brook

618 Institute for Advanced Computational Science (iACS). Work at Pacific Northwest National

619 Laboratory was supported by the Joint Center for Energy Storage Research (JCESR), an Energy

620 Innovation Hub funded by the U.S. Department of Energy, Office of Science, Office of Basic

621 Energy Sciences. 


\section{AUTHOR CONTRIBUTIONS}

623 RA developed the automated NMR framework and the underlying Python-based codes, performed

624 all the necessary calculations, and had primary writing responsibilities. YC and KSH carried the

625 NMR experiments. VM and KTM guided the experimental aspect of the project. NNR guided and 626 led all the computational aspects of the project. All authors contributed to writing and reviewing

627 the manuscript.

\section{COMPETING INTERESTS}

629 The authors declare no competing interests.

630 ADDITIONAL INFORMATION

631 Supplementary information:

632 Correspondence:

633 Correspondence to Nav Nidhi Rajput.

\section{REFERENCES}

1 Zhao, E. W. et al. In situ NMR metrology reveals reaction mechanisms in redox flow batteries. Nature 579, 224-228 (2020).

2 Kim, H. S. et al. Structure and compatibility of a magnesium electrolyte with a sulphur cathode. Nature communications 2, 1-6 (2011).

3 Weber, R. et al. Long cycle life and dendrite-free lithium morphology in anode-free lithium pouch cells enabled by a dual-salt liquid electrolyte. Nature Energy 4, 683-689 (2019).

4 Korb, J.-P., Louis-Joseph, A. \& Benamsili, L. s. Probing structure and dynamics of bulk and confined crude oils by multiscale NMR spectroscopy, diffusometry, and relaxometry. The Journal of Physical Chemistry B 117, $7002-7014$ (2013). 
5 Van de Velde, F., Knutsen, S., Usov, A., Rollema, H. \& Cerezo, A. 1H and 13C high resolution NMR spectroscopy of carrageenans: application in research and industry. Trends in Food Science \& Technology 13, 73-92 (2002).

6 Shimada, I., Ueda, T., Kofuku, Y., Eddy, M. T. \& Wüthrich, K. GPCR drug discovery: integrating solution NMR data with crystal and cryo-EM structures. Nature Reviews Drug Discovery 18, 59-82 (2019).

7 Gorgulla, C. et al. An open-source drug discovery platform enables ultra-large virtual screens. Nature 580, 663-668 (2020).

8 Deng, X. et al. Nuclear magnetic resonance studies of the solvation structures of a highperformance nonaqueous redox flow electrolyte. Journal of Power Sources 308, 172-179 (2016).

9 Cresce, A. V. et al. Solvation behavior of carbonate-based electrolytes in sodium ion batteries. Physical Chemistry Chemical Physics 19, 574-586 (2017).

10 Wang, F. et al. Highly reversible zinc metal anode for aqueous batteries. Nature materials 17, 543-549 (2018).

11 Harks, P., Mulder, F. \& Notten, P. In situ methods for Li-ion battery research: A review of recent developments. Journal of power sources 288, 92-105 (2015).

12 Plewa-Marczewska, A., Kalita, M., Marczewski, M. \& Siekierski, M. NMR studies of equilibriums in electrolytes: Ionic pairing in glymes. Electrochimica Acta 55, 1389-1395 (2010).

13 Barone, V., Improta, R. \& Rega, N. Quantum mechanical computations and spectroscopy: From small rigid molecules in the gas phase to large flexible molecules in solution. Accounts of chemical research 41, 605-616 (2008). 
14 Rajput, N. N., Seguin, T. J., Wood, B. M., Qu, X. \& Persson, K. A. in Modeling Electrochemical Energy Storage at the Atomic Scale 79-124 (Springer, 2018).

15 Hu, J. Z., Jaegers, N. R., Hu, M. Y. \& Mueller, K. T. In situ and ex situ NMR for battery research. Journal of Physics: Condensed Matter 30, 463001 (2018).

16 Dracinsky, M., Möller, H. M. \& Exner, T. E. Conformational sampling by ab initio molecular dynamics simulations improves NMR chemical shift predictions. Journal of chemical theory and computation 9, 3806-3815 (2013).

17 Abella, L., Philips, A. \& Autschbach, J. Ab initio molecular dynamics study of sodium NMR chemical shifts in the methylamine solution of [ $\mathrm{Na}+[2.2 .2]$ cryptand $\mathrm{Na}-$ ]. Physical Chemistry Chemical Physics 23, 339-346 (2021).

18 Casabianca, L. B. \& De Dios, A. C. Ab initio calculations of NMR chemical shifts. The Journal of chemical physics 128, 052201 (2008).

$19 \mathrm{Hu}, \mathrm{M}$. Y. et al. In situ natural abundance $17 \mathrm{O}$ and $25 \mathrm{Mg}$ NMR investigation of aqueous $\mathrm{Mg}(\mathrm{OH}) 2$ dissolution in the presence of supercritical $\mathrm{CO}$. Environmental science \& technology 50, 12373-12384 (2016).

$20 \mathrm{Hu}$, J. Z. et al. $25 \mathrm{Mg} \mathrm{NMR}$ and computational modeling studies of the solvation structures and molecular dynamics in magnesium based liquid electrolytes. Nano energy 46, 436-446 (2018).

21 Yesiltepe, Y. et al. An automated framework for NMR chemical shift calculations of small organic molecules. Journal of cheminformatics 10, 1-16 (2018).

22 Gao, P., Zhang, J., Peng, Q., Zhang, J. \& Glezakou, V.-A. General protocol for the accurate prediction of molecular 13C/1H NMR chemical shifts via machine learning augmented DFT. Journal of Chemical Information and Modeling 60, 3746-3754 (2020). 
23 Chen, Y. et al. Role of Solvent Rearrangement on $\mathrm{Mg} 2+$ Solvation Structures in Dimethoxyethane Solutions using Multimodal NMR Analysis. The Journal of Physical Chemistry Letters 11, 6443-6449 (2020).

24 Wan, C. et al. Natural abundance 17O, 6Li NMR and molecular modeling studies of the solvation structures of lithium bis (fluorosulfonyl) imide/1, 2-dimethoxyethane liquid electrolytes. Journal of Power Sources 307, 231-243 (2016).

25 Rajput, N. N., Qu, X., Sa, N., Burrell, A. K. \& Persson, K. A. The coupling between stability and ion pair formation in magnesium electrolytes from first-principles quantum mechanics and classical molecular dynamics. Journal of the American Chemical Society 137, 3411-3420 (2015).

26 Salama, M. et al. Unique behavior of dimethoxyethane (DME)/Mg (N (SO2CF3) 2) 2 solutions. The Journal of Physical Chemistry C 120, 19586-19594 (2016).

27 SDBSWeb, <https://sdbs.db.aist.go.jp〉.

28 Fulmer, G. R. et al. NMR chemical shifts of trace impurities: common laboratory solvents, organics, and gases in deuterated solvents relevant to the organometallic chemist. Organometallics 29, 2176-2179 (2010).

29 MongoDB Inc., M., 2014.

30 Ong, S. P. et al. Python Materials Genomics (pymatgen): A robust, open-source python library for materials analysis. Computational Materials Science 68, 314-319 (2013).

31 Jain, A. et al. FireWorks: a dynamic workflow system designed for high-throughput applications. Concurrency and Computation: Practice and Experience 27, 5037-5059 (2015).

32 Custodian, <https://github.com/materialsproject/custodian〉. 
33 Gaussian 16 Rev. C.01 (Wallingford, CT, 2016).

34 Plimpton, S. Fast parallel algorithms for short-range molecular dynamics. Journal of computational physics 117, 1-19 (1995).

35 O'Boyle, N. M. et al. Open Babel: An open chemical toolbox. Journal of cheminformatics 3, 33 (2011).

36 Wang, J., Wolf, R. M., Caldwell, J. W., Kollman, P. A. \& Case, D. A. Development and testing of a general amber force field. Journal of computational chemistry 25, 1157-1174 (2004).

37 Halgren, T. A. Merck molecular force field. I. Basis, form, scope, parameterization, and performance of MMFF94. Journal of computational chemistry 17, 490-519 (1996).

38 Landrum, G. RDKit: Open-Source Cheminformatics Software. (2016).

39 Chai, J.-D. \& Head-Gordon, M. Systematic optimization of long-range corrected hybrid density functionals. The Journal of chemical physics 128, 084106 (2008).

40 Rajput, N. N. et al. Elucidating the solvation structure and dynamics of lithium polysulfides resulting from competitive salt and solvent interactions. Chemistry of Materials 29, 33753379 (2017).

41 Blau, S., Spotte-Smith, E., Wood, B., Dwaraknath, S. \& Persson, K. Accurate, Automated Density Functional Theory for Complex Molecules Using On-the-fly Error Correction. (2020).

42 Fadel, E. R. et al. Role of solvent-anion charge transfer in oxidative degradation of battery electrolytes. Nature communications 10, 1-10 (2019). 
43 Borodin, O., Behl, W. \& Jow, T. R. Oxidative stability and initial decomposition reactions of carbonate, sulfone, and alkyl phosphate-based electrolytes. The Journal of Physical Chemistry C 117, 8661-8682 (2013).

44 Jorgensen, W. L., Maxwell, D. S. \& Tirado-Rives, J. Development and testing of the OPLS all-atom force field on conformational energetics and properties of organic liquids. Journal of the American Chemical Society 118, 11225-11236 (1996).

45 Lamoureux, G. \& Roux, B. Modeling induced polarization with classical Drude oscillators: Theory and molecular dynamics simulation algorithm. The Journal of chemical physics 119, 3025-3039 (2003).

46 Kubisiak, P. \& Eilmes, A. Solvation of Mg2+ Ions in Mg (TFSI) 2-Dimethoxyethane Electrolytes-A View from Molecular Dynamics Simulations. The Journal of Physical Chemistry C 122, 12615-12622 (2018).

47 Miertuš, S., Scrocco, E. \& Tomasi, J. Electrostatic interaction of a solute with a continuum. $\mathrm{A}$ direct utilizaion of $\mathrm{AB}$ initio molecular potentials for the prevision of solvent effects. Chemical Physics 55, 117-129 (1981).

48 Miertus, S. \& Tomasi, J. Approximate evaluations of the electrostatic free energy and internal energy changes in solution processes. Chemical physics 65, 239-245 (1982).

49 Pascual-ahuir, J.-L., Silla, E. \& Tunon, I. GEPOL: An improved description of molecular surfaces. III. A new algorithm for the computation of a solvent-excluding surface. Journal of Computational Chemistry 15, 1127-1138 (1994).

50 Beck, A. D. Density-functional thermochemistry. III. The role of exact exchange. J. Chem. Phys 98, 5648-5646 (1993). 
51 Zhao, Y. \& Truhlar, D. G. The M06 suite of density functionals for main group thermochemistry, thermochemical kinetics, noncovalent interactions, excited states, and transition elements: two new functionals and systematic testing of four M06-class functionals and 12 other functionals. Theoretical chemistry accounts 120, 215-241 (2008).

52 Adamo, C. \& Barone, V. Toward reliable density functional methods without adjustable parameters: The PBE0 model. The Journal of chemical physics 110, 6158-6170 (1999).

53 Weigend, F. \& Ahlrichs, R. Balanced basis sets of split valence, triple zeta valence and quadruple zeta valence quality for $\mathrm{H}$ to Rn: Design and assessment of accuracy. Physical Chemistry Chemical Physics 7, 3297-3305 (2005).

54 Marenich, A. V., Cramer, C. J. \& Truhlar, D. G. Universal solvation model based on solute electron density and on a continuum model of the solvent defined by the bulk dielectric constant and atomic surface tensions. The Journal of Physical Chemistry B 113, 6378-6396 (2009).

55 Andersen, A. et al. Structure and dynamics of polysulfide clusters in a nonaqueous solvent mixture of 1, 3-dioxolane and 1, 2-dimethoxyethane. Chemistry of Materials 31, 23082319 (2019).

56 Martínez, L., Andrade, R., Birgin, E. G. \& Martínez, J. M. PACKMOL: a package for building initial configurations for molecular dynamics simulations. Journal of computational chemistry 30, 2157-2164 (2009).

57 Wang, J., Wang, W., Kollman, P. A. \& Case, D. A. Automatic atom type and bond type perception in molecular mechanical calculations. Journal of molecular graphics and modelling 25, 247-260 (2006). 
58 Aqvist, J. Ion-water interaction potentials derived from free energy perturbation simulations. The Journal of Physical Chemistry 94, 8021-8024 (1990).

59 Canongia Lopes, J. N. \& Pádua, A. A. Molecular force field for ionic liquids composed of triflate or bistriflylimide anions. The Journal of Physical Chemistry B 108, 16893-16898 (2004).

60 Köddermann, T., Paschek, D. \& Ludwig, R. Molecular dynamic simulations of ionic liquids: A reliable description of structure, thermodynamics and dynamics. ChemPhysChem 8, 2464-2470 (2007).

61 Anderson, P. M. \& Wilson*, M. R. Developing a force field for simulation of poly (ethylene oxide) based upon ab initio calculations of 1, 2-dimethoxyethane. Molecular Physics 103, 89-97 (2005).

62 Dequidt, A., Devemy, J. \& Padua, A. A. Thermalized Drude oscillators with the LAMMPS molecular dynamics simulator. Journal of chemical information and modeling 56, 260-268 (2016).

63 Borodin, O. Polarizable force field development and molecular dynamics simulations of ionic liquids. The Journal of Physical Chemistry B 113, 11463-11478 (2009).

64 Borodin, O. \& Smith, G. D. Development of quantum chemistry-based force fields for poly (ethylene oxide) with many-body polarization interactions. The Journal of Physical Chemistry B 107, 6801-6812 (2003).

65 Shi, Y. et al. Polarizable atomic multipole-based AMOEBA force field for proteins. Journal of chemical theory and computation 9, 4046-4063 (2013).

66 Hockney, R. W. \& Eastwood, J. W. Computer simulation using particles. (crc Press, 1988). 
67 Thole, B. T. Molecular polarizabilities calculated with a modified dipole interaction. Chemical Physics 59, 341-350 (1981).

68 Borodin, O. et al. Insights into the structure and transport of the lithium, sodium, magnesium, and zinc bis (trifluoromethansulfonyl) imide salts in ionic liquids. The Journal of Physical Chemistry C 122, 20108-20121 (2018).

69 Grimme, S., Antony, J., Ehrlich, S. \& Krieg, H. A consistent and accurate ab initio parametrization of density functional dispersion correction (DFT-D) for the 94 elements H-Pu. The Journal of chemical physics 132, 154104 (2010).

70 Ditchfield, R. Self-consistent perturbation theory of diamagnetism: I. A gauge-invariant LCAO method for NMR chemical shifts. Molecular Physics 27, 789-807 (1974).

71 Wolinski, K., Hinton, J. F. \& Pulay, P. Efficient implementation of the gauge-independent atomic orbital method for NMR chemical shift calculations. Journal of the American Chemical Society 112, 8251-8260 (1990). 


\section{Supplementary Files}

This is a list of supplementary files associated with this preprint. Click to download.

- SIAnautomtedframeworkforhighthroughputpredictionsofNMRchemicalshiftswithinliquidsolutions.pdf

- flatRajputepc.pdf 